\title{
On a generic property of geodesic flows
}

\author{
Hans-Bert Rademacher
}

December 17, 1992

\section{Introduction}

In this paper we are concerned with a sufficient condition for a Riemannian metric on a compact simply-connected manifold to have infinitely many geometrically distinct closed geodesics.

1969 Gromoll-Meyer proved in [GM] that for every Riemannian metric on a compact simply-connected manifold $M$ there are infinitely many geometrically distinct closed geodesics if the sequence $\left(b_{i}(\Lambda M ; F)\right)_{i}$ of Betti numbers of the free loop space $\Lambda M$ is unbounded for some field $F$. Using the theory of minimal models Vigué-Poirrier/Sullivan proved that the rational cohomology algebra $H^{*}(M ; \mathbb{Q})$ of $M$ is generated by a single element if and only if the sequence $\left(b_{i}(\Lambda M ; \mathbb{Q})\right)_{i}$ of Betti numbers of the free loop space $\Lambda M$ of $M$ is bounded. It is a conjecture that the same statement holds for all fields of prime characteristic, partial results are due to McCleary-Ziller [MZ] and Halperin/Vigué-Poirrier [HV].

Now we turn to the manifolds for which the hypothesis of GromollMeyer's theorem does not hold, for example spheres and projective spaces. Then stability properties of the closed geodesics become important. A closed geodesic is hyperbolic if the linearized Poincaré map has no eigenvalue of norm 1. From the bumpy metrics theorem due to Abraham [Ab] and Anosov [An2] and from a pertubation result due to Klingenberg-Takens [KT] one can conclude: A $C^{4}$-generic metric on a compact manifold has either a non-hyperbolic closed geodesic of twist type or all closed geodesics are hyperbolic. In the first case there are infinitely many geometrically distinct closed geodesics in every tubular neighborhood of the closed geodesic of twist type due to a theorem by Moser [Mo]. In the second case there are infinitely many closed geodesics if $M$ is simply-connected due to results by Hingston [Hi] and the author [Ra1]. Hence it follows from these results that a $C^{4}$-generic metric on a compact simply-connected manifold has infinitely many geometrically distinct closed geodesics.

We remark that it is an open question whether there is a Riemannian metric on a simply-connected compact manifold all of whose closed geodesics are hyperbolic. In [Ra2] we show that the examples of metrics on the 2- 
sphere with ergodic geodesic flow given by Donnay and Burns-Gerber provide examples of metrics all of whose homologically visible closed geodesics are hyperbolic.

A closed geodesic $c$ is prime if it is not the iterate of a shorter closed geodesic. The linearized Poincaré map $P_{c}$ of $c$ is a linear symplectic map. If $c$ is not hyperbolic we denote by $z_{j}= \pm \exp \left(2 \pi i \lambda_{j}\right), \lambda_{j} \in[0,1 / 2], j=1, \ldots, l$ the eigenvalues of $P_{c}$ of norm 1 . We call the numbers $0 \leq \lambda_{1}<\ldots<\lambda_{l} \leq$ $1 / 2$ the Poincaré exponents of $c$. We say that a Riemannian metric is strongly bumpy if all eigenvalues of the linearized Poincaré maps of every prime closed geodesic are simple and if any finite set of the disjoint union of the Poincaré exponents of the prime closed geodesics is algebraically independent.

For a metric with only finitely many geometrically distinct closed geodesics the author proved in [Ra1] a relation between the average indices of these closed geodesics. With the help of the normal form of a linear symplectic map which we discuss in section 3 we show in section 5 that for a strongly bumpy Riemannian metric the average indices depend linearly on the Poincaré exponents. Therefore the relation between the average indices implies that the Poincaré exponents are algebraically dependent. Hence we obtain in 5.7:

Theorem 1.1 A strongly bumpy Riemannian metric on a compact simplyconnected manifold carries infinitely many geometrically distinct closed geodesics.

In section 4 we use a pertubation result from the theory of dynamical systems due to Klingenberg-Takens [KT] to show:

Theorem 1.2 The subset of strongly bumpy metrics on a compact manifold is a residual subset of the set of Riemannian metrics with the strong $C^{r}$ topology, where $2 \leq r \leq \infty$.

Combining both theorems we finally obtain

Theorem 1.3 A $C^{r}$-generic Riemannian metric on a compact simply-connected manifold carries infinitely many geometrically distinct closed geodesics, where $2 \leq r \leq \infty$.

Using ideas of Birkhoff it was recently proved that every Riemannian metric on the 2-sphere carries infinitely many geometrically distinct closed geodesics, cf. Franks [Fr] and Bangert [Ba2]. The methods of the proof are restricted to surfaces.

In contrast to the Riemannian case there are examples of non-symmetric Finsler metrics on spheres and projective spaces due to Katok. Ziller studies the geometry of this examples in [Zi]. We remark that theorem 1.1 also holds for Finsler metrics. In these examples the average indices and the Poincaré 
exponents are algebraically dependent. It follows from results of the author [Ra4] that for dimensions $>2$ in these examples there are always two closed geodesics whose mean average indices $\bar{\alpha}_{c}:=\alpha_{c} /$ length $(c)$ coincide. This can be interpreted as a resonance relation.

Grove introduced the concept of isometry-invariant geodesics, cf. [Gr]. There are rotations on the standard sphere in any dimension with only finitely many geometrically distinct invariant geodesics. Using results from [Ra3] one can obtain results analogous to the above stated theorems on the existence of infinitely many geometrically distinct isometry-invariant geodesics on a simply-connected Riemannian manifold with an isometry of finite order under some assumptions on the fixed point set of the isometry.

The results of this paper are contained in the author's Habilitationsschrift [Ra2].

Acknowledgement: I am grateful to the referee for his comments and suggestions. æ

\section{Local pertubation of the Poincaré map}

Througout the paper $M$ is an $n$-dimensional compact $C^{\infty}$ - manifold with tangent bundle $T M$. For a point $p \in M$ we denote by $T_{p} M$ the tangent space at $p . \mathcal{G}^{r}=\mathcal{G}^{r}(M)$ is the set of $C^{r}$-differentiable Riemannian metrics on $M$ with the strong $C^{r}$-topology. For $2 \leq r<\infty \mathcal{G}^{r}$ carries the structure of an open subset of a Banach space after choosing a metric. For $r=\infty$ it carries the structure of an open subset of a Fréchet space. In particular a residual subset of $\mathcal{G}^{r}$ is dense in $\mathcal{G}^{r}$ by Baire's theorem. We call a property of a metric $C^{r}$-generic if the set of metrics satisfying this property contains a residual subset.

For a Riemannian metric $g$ on $M$ the geodesic flow $\Phi_{g}^{t}: T M \rightarrow T M$ on the tangent bundle is defined by $\Phi_{g}^{t}(v)=\dot{c}_{v}(t)$ where $c_{v}: \mathbb{R} \rightarrow M$ is the uniquely determined geodesic with $\dot{c}_{v}(0)=v$.

Using the Levi-Civita connection on $M$ the double tangent bundle TTM has a splitting

$$
T_{X} T M=T_{X}^{v} T M \oplus T_{X}^{h} T M, Z=Z^{v}+Z^{h}
$$

into the vertical and the horizontal subspaces. If $\tau: T M \rightarrow M$ is the canonical projection then the vertical subspace $T_{X}^{v} T M$ is generated by double tangent vectors $\dot{v}(0)$ where $t \in(-\epsilon, \epsilon) \mapsto v(t) \in T M$ is a curve in $T M$ with $v(0)=X$ and $\tau(v(t))=\tau(X)$ for all $t$. The horizontal subspace $T_{X}^{h} T M$ is generated by double tangent vectors $\dot{v}(0)$ where $t \in(-\epsilon, \epsilon) \mapsto v(t) \in T M$ is a parallel field along the curve $t \in(-\epsilon, \epsilon) \mapsto \tau(v(t)) \in M$.

Both these subspaces can be identified with the tangent space $T_{\tau(X)} M$ at $\tau(X)$. Then for $Y=Y^{h}+Y^{v} ; Z=Z^{h}+Z^{v}$

$$
2 \omega(Y, Z)=g\left(Y^{h}, Z^{v}\right)-g\left(Y^{v}, Z^{h}\right)
$$


defines a symplectic form on $T M$. Together with the Hamiltonian $H$ : $T M \rightarrow \mathbb{R}, H(X)=g(X, X) / 2$ the symplectic form $\omega$ makes $T M$ into a Hamiltonian system. The geodesic flow is the corresponding Hamiltonian flow.

The geodesic flow preserves the metric, hence we can restrict ourselves to the geodesic flow $\phi_{g}^{t}: T_{g}^{1} M \rightarrow T_{g}^{1} M$ on the unit tangent bundle $T_{g}^{1} M:=$ $\{X \in T M \mid g(X, X)=1\}$. Non-trivial closed geodesics on $M$ are in oneto-one correspondance to the periodic flow lines of $\phi_{g}^{t}: T_{g}^{1} M \rightarrow T_{g}^{1} M:$ If $\gamma=\left\{\phi_{g}^{t}(v) \mid t \in[0, a]\right\}, v \in T_{g}^{1} M$ is a periodic flow line of period $a>0$, i.e. $v=\phi_{g}^{a}(v)$, then the projection $c(t)=\tau\left(\phi_{g}^{t}(v)\right), t \in[0, a]$ is a closed geodesic on $M$ of length $a$.

For a periodic flow line $\gamma=\left\{\phi_{g}^{t}(v) \mid t \in[0, a]\right\}$ of period $a>0$ we can define the Poincaré map $\mathcal{P}_{g}(\Sigma, \gamma)$ as follows: One can choose a local hypersurface $\Sigma$ in $T_{g}^{1} M$ through $v$ transversal to $\gamma$ such that there are open neighborhoods $\Sigma_{0}, \Sigma_{a}$ of $v$ in $\Sigma$ and a differentiable mapping $\delta: \Sigma_{0} \rightarrow \mathbb{R}$ with $\delta(v)=a$ such that

$$
\mathcal{P}_{g}(\Sigma, \gamma): \Sigma_{0} \rightarrow \Sigma_{a} ; X \mapsto \phi_{g}^{\delta(X)}(X)
$$

is a diffeomorphism. $\omega$ induces a symplectic form on $\Sigma$ and $\mathcal{P}_{g}(\Sigma, \gamma)$ becomes a symplectic diffeomorphism. Fixed points of $\mathcal{P}_{g}(\Sigma, \gamma)$ correspond to periodic flow lines nearby $\gamma$. Let $V$ be the ortogonal complement of $v$ in $T_{\tau(v)} M$, i.e. $V$ is a $(n-1)$-dimensional vector space. On $V \oplus V$ we have the canonical symplectic form

$$
\eta\left(\left(x_{1}, x_{2}\right),\left(y_{1}, y_{2}\right)\right):=g\left(x_{1}, y_{2}\right)-g\left(x_{2}, y_{1}\right) .
$$

One can choose $\Sigma$ such that the linearized Poincaré map $P_{g}(\gamma):=d \mathcal{P}_{g}(\Sigma, \gamma)(v)$ is a linear symplectic map of $V \oplus V$ and

$$
P_{g}(\gamma)(J(0), \nabla J(0))=(J(a), \nabla J(a)) .
$$

Here $J(t)$ is a normal Jacobi field along the geodesic $c=\tau \circ \gamma$ and $\nabla J(t)$ is the covariant derivative along $c$. After choosing a symplectic basis for $V \oplus V$ we can identify the group of symplectic linear maps of $V \oplus V$ with the symplectic group $\operatorname{Sp}(n-1)$ of $\mathbb{R}^{n-1} \oplus \mathbb{R}^{n-1}$.

With a closed geodesic $c: S^{1}=\mathbb{R} / \mathbb{Z} \rightarrow M$ all iterates $c^{m}: S^{1} \rightarrow$ $M ; c(t)=c(m t)$ for $m \in \mathbb{N}$ are closed geodesics, too. We call a closed geodesic $c$ prime if it is not the iterate of a shorter curve. Analogously we call a periodic flow line $\gamma=\left\{\phi_{g}^{t}(v) \mid t \in[0, a]\right\}$ prime, if $a$ is the minimal period, i.e. $c(t)=\tau \circ \gamma(a t)$ is a prime closed geodesic. We call the closed geodesic $c: S^{1} \rightarrow M$ resp. the periodic flow line $\gamma=\left\{\phi_{g}^{t}(v) \mid t \in[0, a]\right\}$ non-degenerate if 1 is not an eigenvalue of the linearized Poincaré map $P_{c}:=P_{g}(\gamma)$. Then $\gamma$ resp. $c$ is an isolated periodic flow line resp. an isolated closed geodesic. 
A Riemannian manifold is called bumpy if all periodic flow lines of the geodesic flow resp. all closed geodesics are non-degenerate. Since $P_{c^{m}}=$ $\left(P_{c}\right)^{m}$ this is equivalent to the following assumption: All Poincaré exponents of every closed geodesic are irrational, i.e. if $z=\exp (2 \pi i \lambda)$ is an eigenvalue of $P_{c}$, then $\lambda \in \mathbb{R}-\mathbb{Q}$.

We will use the following local pertubation argument due to KlingenbergTakens:

Theorem 2.1 [KT, thm.2] Let $\gamma=\left\{\phi_{g_{0}}^{t}(v)\right\}$ be a periodic flow line of period a of the geodesic flow $\left(\phi_{g_{0}}^{t}\right)$ of the metric $g_{0} \in \mathcal{G}^{r}, r \geq 2$. Let $W$ be an open neighborhood of the point $\tau(v) \in M$ on $M$. Denote by $\mathcal{G}^{r}\left(\gamma, g_{0}, W\right)$ the set of metrics $g \in \mathcal{G}^{r}$, for which $\gamma$ is a periodic flow line of period $a>0$ and such that the support of $g-g_{0}$ lies in $W$.

If $Q$ is an open and dense invariant subset of the symplectic group $\operatorname{Sp}(n-1)$ then there is for every neighborhood $\mathcal{V}$ of $g_{0}$ in $\mathcal{G}^{r}$ a metric $g \in$ $\mathcal{V} \cup \mathcal{G}^{r}\left(\gamma, g_{0}, W\right)$ such that $P_{g}(\gamma) \in Q$.

Let $\mathcal{G}_{b}^{r} \subset \mathcal{G}^{r}$ be the subset of bumpy metrics. For $t>0$ let $\mathcal{G}_{b}^{r}(t)$ be the subset of metrics such that every periodic flow line of period $\leq t$ is nondegenerate. Hence this is equivalent to the following assumption: If $\gamma$ is a prime periodic flow line of period $a$ and if $\lambda$ is a Poincaré exponent of $\gamma$ then $\lambda^{k} \neq 1$ for all $k \in \mathbb{N}, k \leq t / a$. In particular there are only finitely many periodic flow lines of period $\leq t$.

Abraham formulated the bumpy metrics theorem in [Ab], a complete proof was given by Anosov in [An2]. It follows from the

Lemma 2.2 $[\mathrm{An} 2, \S 4]$ a) $\mathcal{G}_{b}^{r}(t)$ is an open subset of $\mathcal{G}^{r}$.

b) $\mathcal{G}_{b}^{r}(t)$ is a dense subset of $\mathcal{G}^{r}$.

For the proof of part b) one uses the local pertubation theorem 2.1 by Klingenberg-Takens and a transversality theorem due to Abraham. Since

$$
\mathcal{G}_{b}^{r}=\bigcap_{k=1}^{\infty} \mathcal{G}_{b}^{r}(k)
$$

the lemma implies the

Theorem 2.3 (bumpy metrics theorem) [Ab] [An2] For $2 \leq r \leq \infty$ the set $\mathcal{G}_{b}^{r}$ of bumpy metrics is a residual subset of the set $\mathcal{G}^{r}$ of metrics with the strong $C^{r}$-topology on a compact manifold.

One also says for short: For $2 \leq r \leq \infty$ a $C^{r}$-generic metric on a compact manifold is bumpy.

In the proof of part b) of lemma 2.2 the following lemma is used. We formulate it here since we will use it in the sequel: 
Lemma 2.4 [An2, §4] Let $g_{0} \in \mathcal{G}_{b}^{r}(t), 2 \leq r \leq \infty$ and let $\left\{\phi_{g_{0}}^{t}\left(v_{1}\right)\right\}, \ldots$, $\left\{\phi_{g_{0}}^{t}\left(v_{N}\right)\right\}$ be the prime periodic flow lines of the geodesic flow with periods $a_{1}, \ldots, a_{N} \in(0, t]$. Then there is an open neighborhood $\mathcal{U}$ of $g_{0}$ in $\mathcal{G}^{r}$ and there are continuous maps $v_{k}: \mathcal{U} \rightarrow T M, a_{k}: \mathcal{U} \rightarrow \mathbb{R}^{+}, k=1, \ldots, N$ such that $v_{k}\left(g_{0}\right)=v_{k}, a_{k}\left(g_{0}\right)=a_{k}$ with the following property: For every $g \in \mathcal{U}\left\{\phi_{g}^{t}\left(v_{1}(g)\right)\right\}, \ldots,\left\{\phi_{g}^{t}\left(v_{N}(g)\right)\right\}$ are the prime periodic flow lines of $\phi_{g}^{t}:$ $T_{g}^{1} M \rightarrow T_{g}^{1} M$ with periods $\left.\left\{a_{1}(g), \ldots, a_{N}(g)\right)\right\}$ and there are no other prime periodic flow lines of $\phi_{g}^{t}$ with period $\leq t$.

$æ$

\section{Normal form of symplectic maps}

Normal forms for symplectic linear mappings were discussed by several authors. We use the notation introduced by Ballmann-Thorbergsson-Ziller [BTZ]. We consider a finite-dimensional real vector space $E$ with symplectic form $\omega$. Let $k=2 n$ be the even dimension of $E$. A linear endomorphism $P$ of $E$ is called symplectic, if it preserves $\omega$. $\omega$ induces a non-degenerate Hermitian form $\omega_{h}$ on the complexification $E_{\mathbb{C}}$ of $E$ :

$\omega_{h}\left(X_{1}+i Y_{1}, X_{2}+i Y_{2}\right)=\omega\left(X_{1}, Y_{2}\right)-\omega\left(Y_{1}, X_{2}\right)+i\left(\omega\left(X_{1}, X_{2}\right)+\omega\left(Y_{1}, Y_{2}\right)\right)$.

We also denote by $P$ the canonical linear extension of $P$ onto $E_{\mathbb{C}}$. Then $P$ also preserves $\omega_{h}$. For an eigenvalue $z \in \mathbb{C}$ of $P$ we denote by $V(z):=\operatorname{ker} N_{z}^{k}$ the generalized eigenspace, here $N_{z}=P z^{-1}-\mathrm{id}$. Let $d(P, z):=\operatorname{dim} N_{z}^{k}$. We denote by $\operatorname{Spec}(P)$ the set of eigenvalues, if $z$ is an eigenvalue then also $z^{-1}, \bar{z}, \bar{z}^{-1}$.

$$
E_{\mathbb{C}}=\bigoplus_{z \in \text { Spec },|z|=1} V(z) \oplus \bigoplus_{z \in \text { Spec },|z|>1}\left(V(z) \oplus V\left(z^{-1}\right)\right)
$$

is a decomposition of $E_{\mathbb{C}}$ in $P$-invariant pairwise orthogonal non-degenerate subspaces. For $m \in \mathbb{N}$ we denote by $J(z, m)$ a $P$-invariant non-degenerate subspace of $E_{\mathbb{C}}$ with the following property: There is a vector $X \in J(z, m)$ such that $N_{z}^{m}(X)=0, \omega_{h}\left(N_{z}^{m-1}(X), X\right)=0$ and $X, N_{z}(X), \ldots, N_{z}^{m-1}(X)$ is a basis of $J(z, m)$. Then there is a splitting of $V(z)$ in pairwise orthogonal non-degenerate $P$-invariant subspaces: If $|z|=1$ then

$$
V(z)=\bigoplus_{j=1}^{j(z)} J\left(z, m_{j}\right),
$$

if $|z|>1$ then

$$
V(z)=\bigoplus_{j=1}^{j(z)}\left(J\left(z, m_{j}\right) \oplus J\left(\bar{z}^{-1}, m_{j}\right)\right) .
$$


One can also define corresponding real Jordan blocks. A real Jordan block $J_{\mathbb{R}}\left(z, m_{j}\right)$ is a minimal $P$-invariant non-degenerate subspace of $E$. There is a further symplectic invariant for a Jordan block $J\left(z, m_{j}\right)$ resp. $J_{\mathbb{R}}\left(z, m_{j}\right)$. This symplectic invariant $\sigma_{j} \in\{0, \pm 1\}$ is called the sign, we denote the corresponding Jordan space by $J\left(z, m_{j}, \sigma_{j}\right)$ resp. $J_{\mathbb{R}}\left(z, m_{j}, \sigma_{j}\right)$. We set $\sigma_{j}=$ 0 if $|z|>1$. The sign of $J\left(z, m_{j}\right)$ is non-zero if $|z|=1$. If $|z|=1$ and $m_{j}=$ $2 l-1$ (resp. $m_{j}=2 l$ ) and if $X \in J\left(z, m_{j}\right)$ satisfies $N_{z}^{m_{j}-1} X \neq 0$ then $\sigma_{j} \in$ $\{ \pm 1\}$ is defined by $-\sigma_{j} \omega_{h}\left(N_{z}^{l-1} X, N_{z}^{l-1} X\right)>0\left(\operatorname{resp.} i \sigma_{j}\left(N_{z}^{l} X, N_{z}^{l-1} X\right)>\right.$ $0)$. If $\operatorname{Im} z>0$ then the sign of $J_{\mathbb{R}}\left(z, m_{j}\right)$ equals the sign of $J\left(z, m_{j}\right)$. If $z= \pm 1$ then the sign of $J_{\mathbb{R}}\left(z, m_{j}\right)$ equals the sign of $J\left(z, m_{j}\right)$ if $m_{j}$ is even, otherwise the sign is 0 . So finally we obtain a splitting

$$
E=\bigoplus_{\operatorname{Im} z \geq 0,|z| \geq 1} \bigoplus_{j=1}^{j(z)} J_{\mathbb{R}}\left(z, m_{j}, \sigma_{j}\right)
$$

of $E$ into pairwise orthogonal minimal $P$-invariant non-degenerate subspaces. The sequence $\left(z, m_{j}, \sigma_{j}\right), z \in \operatorname{Spec}(P),|z| \geq 1, \operatorname{Im} z \geq 0$ determines - up to order - uniquely the conjugacy class of $P$ in the group of symplectic linear maps of $E$.

$æ$ After choosing a symplectic basis for $E$ we obtain a symplectic isomorphism of $E$ with $\mathbb{R}^{n} \oplus \mathbb{R}^{n}$ with its canonical symplectic form

$$
\omega\left(\left(x_{1}, x_{2}\right),\left(y_{1}, y_{2}\right)\right)=\left\langle x_{1}, y_{2}\right\rangle-\left\langle x_{2}, y_{1}\right\rangle .
$$

Hence we can identify the symplectic group of $E$ with the symplectic group

$$
\operatorname{Sp}(n)=\left\{P \in \operatorname{Gl}(2 n ; \mathbb{R}) \mid \omega(P x, P y)=\omega(x, y) \forall x, y \in \mathbb{R}^{2 n}\right\}
$$

of linear symplectic maps of $\mathbb{R}^{2 n}$. Again we also denote by $P$ the canonical complex linear extension $P: \mathbb{C}^{n} \oplus \mathbb{C}^{n} \rightarrow \mathbb{C}^{n} \oplus \mathbb{C}^{n}$. For $z \in \mathbb{C}$ let $d(P, z)=$ $\operatorname{dim}(P-z \text { id })^{2 n}$, i.e. $d(P, z)$ is the dimension of the generalized eigenspace of $z$.

Definition 3.1 We denote by

$$
\widetilde{\mathrm{Sp}}(n):=\{P \in \operatorname{Sp}(n) \mid d(P, 1)=d(P,-1)=0\}
$$

the subset of symplectic matrices $P$ for which neither 1 nor -1 is an eigenvalue. Let

$$
\mathrm{Sp}^{*}(n):=\{P \in \widetilde{\mathrm{Sp}} \mid \forall z \in \mathbb{C}: d(P, z) \leq 1\},
$$

i.e. $P \in \mathrm{Sp}^{*}(n)$ has only simple eigenvalues.

Proposition 3.2 The subset $\mathrm{Sp}^{*}(n)$ is an open and dense subset of $\operatorname{Sp}(n)$. 
Proof. A symplectic map $P \in \mathrm{Sp}(n)$ lies in $\mathrm{Sp}^{*}(n)$ iff the discriminant $D(\chi(P))$ of the characteristic polynomial $\chi(P)$ does not vanish. Hence $\mathrm{Sp}^{*}(n)$ is an open subset of $\operatorname{Sp}(n)$. Now assume that $\mathrm{Sp}^{*}(n)$ is not dense in $\operatorname{Sp}(n)$. Then there is a non-empty open subset $U \subset \operatorname{Sp}(n)$ such that $U \cap \operatorname{Sp}^{*}(n)=\emptyset$. The function $F: P \in \operatorname{Sp}(n) \mapsto D(\chi(P)) \in \mathbb{R}$ is analytic. By assumption $F$ vanishes on the open non-empty subset $U \subset \operatorname{Sp}(n)$. Hence it vanishes identically on $\operatorname{Sp}(n)$, which is a contradiction.

For $l \in\{0,1, \ldots, n\}$ we define the invariant subset

$$
\operatorname{Sp}(l ; n):=\left\{P \in \mathrm{Sp}^{*}(n) \mid \sum_{|z|=1, \operatorname{Im} z>0} d(P, z)=l\right\} .
$$

Since for all $P \in \operatorname{Sp}^{*}(n)$ we have $d(P, z) \leq 1$ and since for $z \notin \mathbb{R},|z| \neq 1$ with $z$ also $z^{-1}, \bar{z}, \bar{z}^{-1}$ are pairwise distinct eigenvalues of $P$ we obtain:

$\mathrm{Sp}(l ; n)$ is an open subset of $\mathrm{Sp}^{*}(n)$ and

$$
\mathrm{Sp}^{*}(n)=\bigcup_{l=0}^{n} \operatorname{Sp}(l ; n) .
$$

The possible normal forms of $P \in \operatorname{Sp}(l ; n)$ are as follows: Let $z_{j}=\exp \left(2 \pi i \lambda_{j}\right)$, $j=1, \ldots, l ; 0<\lambda_{1}<\ldots<\lambda_{l}<1 / 2$ be the eigenvalues of $P$ with $\left|z_{j}\right|=1$. We call the numbers $\lambda_{j} \in(0,1 / 2)$ the Poincaré exponents of $P$. Let $z_{j}: j=l+1, \ldots, n$ be the eigenvalues with $\operatorname{Im}\left(z_{j}\right) \geq 0$ and $\left|z_{j}\right|>1$. Then only Jordan blocks with $m_{j}=1$ can occur since all eigenvalues are simple. Hence there is a splitting

$$
\mathbb{R}^{2 n}=\bigoplus_{j=1}^{m} J_{\mathbb{R}}\left(z_{j}, 1, \sigma_{j}\right)
$$

with $\sigma_{j} \in\{1,-1\}$ for $j \leq l$ and $\sigma_{j}=0$ for $j \geq l+1$. With respect to a symplectic basis $P \mid J_{\mathbb{R}}\left(z_{j}, 1, \sigma_{j}\right)$ has the following matrix representation:

a) If $z_{j}=\exp \left(2 \pi i \lambda_{j}\right)$, i.e. $j \in\{1, \ldots, l\}, \sigma_{j} \in\{1,-1\}$ :

$$
P \mid J_{\mathbb{R}}\left(z_{j}, 1, \sigma_{j}\right)=\left(\begin{array}{cc}
\cos \left(2 \pi \lambda_{j}\right) & -\sigma_{j} \sin \left(2 \pi \lambda_{j}\right) \\
\sigma_{j} \sin \left(2 \pi \lambda_{j}\right) & \cos \left(2 \pi \lambda_{j}\right)
\end{array}\right) .
$$

b) If $\left|z_{j}\right|>1$ and $\operatorname{Im}\left(z_{j}\right)=0$, then

$$
P \mid J_{\mathbb{R}}\left(z_{j}, 1,0\right)=\left(\begin{array}{cc}
z_{j} & 0 \\
0 & z_{j}^{-1}
\end{array}\right) .
$$

c) If $\left|z_{j}\right|>1$ and $\operatorname{Im}\left(z_{j}\right)>0$, then

$$
P \mid J_{\mathbb{R}}\left(z_{j}, 1,0\right)=\left(\begin{array}{cccc}
z_{j} & 0 & 0 & 0 \\
0 & z_{j}^{-1} & 0 & 0 \\
0 & 0 & \bar{z}_{j} & 0 \\
0 & 0 & 0 & \bar{z}_{j}^{-1}
\end{array}\right) .
$$


In the following lemma we show how one can define open and dense subsets of $\operatorname{Sp}(l ; n)$ by polynomial relations between the Poincaré exponents. We introduce the analytic mapping

$$
f: \operatorname{Sp}(l ; n) \rightarrow\left(0, \frac{1}{2}\right)^{l}, f(P)=\left(\lambda_{1}(P), \ldots, \lambda_{l}(P)\right) .
$$

Here $\lambda_{1}(P)<\ldots<\lambda_{l}(P)<1 / 2$ are the Poincaré exponents of $P$.

Lemma 3.3 Let $p$ be a non-trivial polynomial in $l$ variables with real coefficients. Then

$$
Q(p):=\left\{\left(x_{1}, \ldots, x_{l}\right) \in \mathbb{R}^{l} \mid p\left(x_{1}, \ldots, x_{l}\right) \neq 0\right\}
$$

is open and dense in $\mathbb{R}^{l}$ and its preimage

$$
f^{-1}(Q(p)):=\left\{P \in \operatorname{Sp}(l ; n) \mid p\left(\lambda_{1}(P), \ldots, \lambda_{l}(P)\right) \neq 0\right\}
$$

under $f$ is an open and dense invariant subset of $\operatorname{Sp}(l ; n)$.

Proof. $Q(p)$ is the complement of an algebraic set, hence it is open and dense in $\mathbb{R}^{l}$ and $f^{-1}(Q(p))$ is open in $\operatorname{Sp}(l ; n)$. Let $A$ be a connected component of $\operatorname{Sp}(l ; n)$, hence it is an open and non-empty subset of $\operatorname{Sp}(l ; n)$. Then $f^{-1}(Q(p)) \cap A$ is a non-empty open subset of $A$ since $p$ is non-trivial. If $f^{-1}(Q(p)) \cap A$ is not a dense subset of $A$ then there is an open non-empty subset $U \subset A$ with $p(f(U))=\{0\}$. But since $f$ is analytic this would imply that $p \circ f$ vanishes on $A$, which is a contradiction.

æ

\section{A generic metric is strongly bumpy}

In section 3 we defined the invariant subset $\operatorname{Sp}^{*}(n-1)$ of linear symplectic maps with only simple eigenvalues. Let $\mathcal{G}^{*}(t)$ be the subset of all metrics in $\mathcal{G}^{r}$ such that for every periodic flow line of period $\leq t$ we have $P_{g}(\lambda) \in$ $\mathrm{Sp}^{*}(n-1)$. Then by theorem 2.1 the set $\mathcal{G}^{*}(t)$ is open and dense and the set $\mathcal{G}^{*}:=\mathcal{G}^{*}(\infty)$ is a residual subset of $\mathcal{G}$.

If the linearized Poincaré mapping $P_{g}(\gamma)$ does not have any eigenvalue $z$ with $|z|=1$ then we call $\gamma$ resp. the corresponding closed geodesic $c=\tau \circ \gamma$ hyperbolic. If $g \in \mathcal{G}^{*}(t)$ and if the period of $\gamma$ is $\leq t$ then $\gamma$ is hyperbolic iff $P_{g}(\gamma) \in \operatorname{Sp}(0 ; n-1)$.

For $t>0$ and for a metric $g \in \mathcal{G}^{*}(t)$ we define the sequence $\Lambda_{g}(t):=$ $\left(\lambda_{i}(g)\right)_{i=1, \ldots, n(g ; t)}$ of Poincaré exponents as follows: If all periodic flow lines of period $\leq t$ are hyperbolic, then $n(g ; t)=0$. Otherwise let $\left(\gamma_{k}\right)_{k=1, \ldots, m(g ; t)}$ be the non-hyperbolic prime periodic flow lines with periods $\left(a_{k}\right)_{k+1, \ldots, m(g ; t)}$, $0<a_{k} \leq t$. Let $P_{k}:=P_{g}\left(\gamma_{k}\right)$ be the linearized Poincaré map of $\gamma_{k}$, since $g \in$ 
$\mathcal{G}^{*}(t)$ we have $P_{k} \in \mathrm{Sp}^{*}(n-1)$. Hence there is a number $l(k) \in\{1, \ldots, n-1\}$ with $P_{k} \in \mathrm{Sp}^{*}(l(k) ; n-1)$. Let for any $k \in\{1, \ldots, m(g ; t)\}$

$$
0<\lambda_{k, 1}<\ldots<\lambda_{k, l(k)}<\frac{1}{2}
$$

be the Poincaré exponents of $P_{k}$, i.e.

$$
z_{k, 1}= \pm \exp \left(2 \pi i \lambda_{k, 1}\right), \ldots, z_{k, l(k)}= \pm \exp \left(2 \pi i \lambda_{k, l(k)}\right)
$$

are the eigenvalues of $P_{k}$ with norm 1. Then we define the sequence $\Lambda_{g}(t)=$ $\left(\lambda_{1}(g), \ldots, \lambda_{n(g ; t)}(g)\right)$ as follows: Let $L(k):=\sum_{r=1}^{k-1} l(r)$ i.e. $n(g ; t)=$ $L(m(g ; t)+1)$. Then $\lambda_{L(k)+l}(g)=\lambda_{k, l}, 1 \leq l \leq l(k)$ for all $\left.k \in 1, \ldots, m(g ; t)\right\}$. Hence

$$
\begin{aligned}
\Lambda_{g}(t) & =\left(\lambda_{1}(g), \ldots, \lambda_{n(g ; t)}(g)\right) \\
& =\left(\lambda_{1,1}, \ldots, \lambda_{1, l(1)}, \lambda_{2,1}, \ldots, \lambda_{m(g ; t), l(m(g ; t))}\right)
\end{aligned}
$$

If $g \in \mathcal{G}^{*}=\bigcap_{n=1}^{\infty} \mathcal{G}^{*}(n)$, then $\Lambda_{g}=\left(\lambda_{i}\right)_{i=1, \ldots, n(g)}$ is the sequence of Poincaré exponents of $g$. Here $n(g)=0$ resp. $\Lambda_{g}=\emptyset$, if all periodic flow lines are hyperbolic and $n(g)=\infty$ if there are infinitely many non-hyperbolic periodic flow lines.

Let $\mathbb{Z}\left[x_{1}, \ldots, x_{d}\right]^{*}$ be the set of polynomials $p \neq 0$ with $d$ variables and with integer coefficients, i.e.

$$
p=p\left(x_{1}, \ldots, x_{d}\right)=\sum_{0 \leq i_{1}, \ldots, i_{d}} a_{i_{1}, \ldots, i_{d}} x_{1}^{i_{1}} \cdot \ldots \cdot x_{d}^{i_{d}},
$$

$a_{i_{1}, \ldots, i_{d}} \in \mathbb{Z}$.

Definition 4.1 A metric $g \in \mathcal{G}^{*}$ on a compact manifold with sequence $\Lambda_{g}=\left(\lambda_{i}(g)\right)_{i=1, \ldots, n(g)}, n(g) \in \mathbb{N}_{0} \cup\{\infty\}$ of Poincaré exponents is called strongly bumpy if the following holds: Either all periodic flow lines (i.e. all closed geodesics) are hyperbolic (i.e. $n(g)=0$ ) or for all $d \in \mathbb{N}$ with $1 \leq d \leq n(g)$ and for all polynomials $p \in \mathbb{Z}\left[x_{1}, \ldots, x_{d}\right]^{*}$ we have

$$
p\left(\lambda_{1}(g), \ldots, \lambda_{d}(g)\right) \neq 0 .
$$

This means that the Poincaré exponents of $g$ are algebraically independent. Let $\mathcal{G}_{s}=\mathcal{G}_{s}^{r}$ be the set of strongly bumpy metrics in $\mathcal{G}=\mathcal{G}^{r}$.

Now we want to show that the set $\mathcal{G}_{s}$ is residual in $\mathcal{G}^{r}$. We define for $d \in \mathbb{N}, L>0$ the polynomial

$$
p(d, L)=p(d, L)\left(x_{1}, \ldots, x_{d}\right) \in \mathbb{Z}\left[x_{1}, \ldots, x_{d}\right]^{*}
$$

as the product of all polynomials in $\mathbb{Z}\left[x_{1}, \ldots, x_{d}\right]^{*}$ whose degree is at most $L$ and whose coefficients have absolute value at most $L$. This polynomial is 
symmetric in $x_{1}, \ldots, x_{d}$. Then we define the set $\mathcal{G}_{s}(t)$ of metrics $g \in \mathcal{G}^{*}(t)$ for which

$$
p(n(g ; t), t)\left(\lambda_{1}(g), \ldots, \lambda_{n(g ; t)}(g)\right) \neq 0
$$

if $n(g ; t) \geq 1$.

Lemma 4.2 For $2 \leq r \leq \infty$ the set $\mathcal{G}_{s}(t)$ is an open and dense subset of $\mathcal{G}^{r}$.

Proof. a) We first show that $\mathcal{G}_{s}(t)$ is an open subset. If $n\left(g_{0} ; t\right)=0$, i.e. if all prime periodic flow lines with period $\leq t$ are hyperbolic, then there is an open subset $\mathcal{U}$ of $g_{0}$ in $\mathcal{G}^{r}$ with $n(g ; t)=0$ for all $g \in \mathcal{U}$, i.e. $\mathcal{U} \subset \mathcal{G}_{s}(t)$. Now assume $n\left(g_{0} ; t\right) \geq 1$, let $\left\{\phi_{g_{0}}^{t}\left(v_{k}\right)\right\}, k=1, \ldots, m\left(g_{0} ; t\right)$ be the prime non-hyperbolic periodic flow lines of periods $0<a_{k} \leq t$. By lemma 2.4 there is an open neighborhood $\mathcal{U}$ of $g_{0}$ and there are continuous maps $v_{k}: \mathcal{U} \rightarrow T M, a_{k}: \mathcal{U} \rightarrow \mathbb{R}^{+}, k=1, \ldots, m\left(g_{0} ; t\right)$, such that $\gamma_{k}(g)=$ $\left\{\phi_{g}^{t}\left(v_{k}(g)\right)\right\}$ are prime periodic flow lines of $\left(\phi_{g}^{t}\right)$ with periods $a_{k}(g)$ and $\gamma_{k}\left(g_{0}\right)=\gamma_{k}, a_{k}\left(g_{0}\right)=a_{k}, v_{k}\left(g_{0}\right)=v_{k}$. And there are no further prime periodic flow lines of $g \in \mathcal{U}$ with period $\leq t$. The mapping

$$
P_{k}: \mathcal{U} \longrightarrow \mathrm{Sp}(n-1), g \mapsto P_{g}\left(\gamma_{k}\right)
$$

is continuous for every $k \in\left\{1, \ldots, m\left(g_{0} ; t\right)\right\}$ since $r \geq 2$. By definition of $l(k)$ we have $P_{k}\left(g_{0}\right) \in \operatorname{Sp}(l(k) ; n-1) . \operatorname{Sp}(l(k) ; n-1)$ is an open subset of $\operatorname{Sp}^{*}(n-1)$, cf. section 3 , hence there is an open subset $\mathcal{U}_{1} \subset \mathcal{U}$ of $g_{0}$ with $P_{g}\left(\gamma_{k}(g)\right) \in \operatorname{Sp}(l(k) ; n-1)$ for all $g \in \mathcal{U}_{1}$. Let $\lambda_{j}(g), j=1, \ldots, n\left(g_{0} ; t\right)$ be the Poincaré exponents of the prime periodic flow lines $\gamma_{k}(g), k=1, \ldots, m\left(g_{0} ; t\right)$. Here we note that $m\left(g_{0} ; t\right) \geq m(g ; t)$. The map

$$
F_{t}: \mathcal{U}_{1} \rightarrow(0,1 / 2)^{n\left(g_{0} ; t\right)}, F_{t}(g)=\left(\lambda_{1}(g), \ldots, \lambda_{n\left(g_{0} ; t\right)}(g)\right)
$$

is continuous. $g_{0} \in \mathcal{G}_{s}(t)$ means that $p\left(n\left(g_{0}, t\right) ; t\right)\left(F_{t}\left(g_{0}\right)\right) \neq 0$. Hence there is an open neighborhood $\mathcal{U}_{2} \subset \mathcal{U}_{1}$ of $g_{0}$ in $\mathcal{G}^{r}$ with $p\left(n\left(g_{0} ; t\right), t\right)\left(F_{t}(g)\right) \neq 0$ for all $g \in \mathcal{U}_{2}$. The polynomial $p(n(g ; t), t)$ is a factor of the polynomial $p\left(n\left(g_{0} ; t\right), t\right)$ since $n\left(g_{0} ; t\right) \geq n(g ; t)$, hence $\mathcal{U}_{2} \subset \mathcal{G}_{s}(t)$.

b) We show that $\mathcal{G}_{s}(t)$ is a dense subset of $\mathcal{G}^{*}(t)$. This is sufficient since $\mathcal{G}^{*}(t)$ is a dense subset of $\mathcal{G}=\mathcal{G}^{r}$. We start with a metric $g_{0} \in \mathcal{G}^{*}(t)$. If $n\left(g_{0} ; t\right)=0$ then by definition $g_{0} \in \mathcal{G}_{s}(t)$. So we assume $n\left(g_{0} ; t\right) \geq$ 1 , let $\left(\gamma_{k}\right)_{k=1, \ldots, m(t)}$ be the prime periodic flow lines of $\phi_{g_{0}}^{t}$ with periods $\left(a_{k}\right)_{k=1, \ldots, m(t)}, 0<a_{k} \leq t$. Let $P_{k}=P_{g_{0}}\left(\gamma_{k}\right) \in \operatorname{Sp}(l(k) ; n-1)$ be the linearized Poincaré maps and $c_{k}$ the corresponding closed geodesics on $M$. Let $L(k):=\sum_{r=1}^{k-1} l(r)$, i.e. $\lambda_{1}\left(g_{0}\right), \ldots, \lambda_{L(k)}\left(g_{0}\right)$ are the Poincaré exponents of the prime periodic flow lines $\gamma_{1}, \ldots, \gamma_{k}-1$. As in lemma 2.4 choose an open neighborhood $\mathcal{U}$ of $g_{0}$ in $\mathcal{G}^{*}(t)$, hence $\mathcal{U}$ is also open in $\mathcal{G}$. 
We prove by induction over $k \in\{1, \ldots, m(t)\}$ that $\mathcal{U} \cap \mathcal{G}_{s}(t) \neq \emptyset$. This means we perturb in every step a single periodic flow line to obtain a metric in $\mathcal{G}_{s}(t)$.

We choose pairwise disjoint open neighborhoods $W_{k}$ on the manifold $M$ of points $p_{k}=\tau\left(\phi_{g_{0}}^{t_{k}}\left(v_{k}\right)\right)=c_{k}\left(t_{k}\right)$ on the closed geodesics $c_{k}, k=$ $1, \ldots, m(t)$ such that the geodesics $c_{j}, j \neq k$ do not meet $W_{k}$. For an element $P \in \mathrm{Sp}(l ; n-1)$ we denote by

$$
0<\lambda_{1}(P)<\cdots<\lambda_{l}(P)<\frac{1}{2}
$$

the Poincaré exponents, i.e. $z_{j}=\exp \left(2 \pi i \lambda_{j}(P)\right)$ are the eigenvalues of $P$ on the unit circle. We conclude from lemma 3.3 that the invariant subset

$$
\begin{aligned}
Q_{1}:= & \{P \in \operatorname{Sp}(n-1) \mid P \in \operatorname{Sp}(l(1) ; n-1) \Rightarrow \\
& \left.p(l(1), t)\left(\lambda_{1}(P), \ldots, \lambda_{l(1)}(P)\right) \neq 0\right\}
\end{aligned}
$$

of $\operatorname{Sp}(n-1)$ is an open and dense subset. It follows from the local pertubation theorem 2.1 that there is a metric $g_{1} \in \mathcal{U} \cap \mathcal{G}\left(\gamma_{1}, g_{0}, W_{1}\right)$ with $P_{g_{1}}\left(\gamma_{1}\right) \in$ $\operatorname{Sp}(l(1) ; n-1) \cap Q_{1}$. Hence $g_{1}$ has the same periodic flow lines $\left\{\gamma_{k}\right\}_{k=1, \ldots, m(t)}$ with the same periods $\left\{a_{k}\right\}_{k=1, \ldots, m(t)}$ as $g_{0}$. The support of the pertubation $g_{1}-g_{0}$ lies in $W_{1}$, i.e. the metrics $g_{0}, g_{1}$ coincide in neighborhoods of the other closed geodesics $c_{2}, \ldots, c_{m(t)}$.

Now assume that there is a metric $g_{k-1} \in \mathcal{G}^{*}(t) \cap \mathcal{U}$ for $k \geq 2$ with the following properties: the periodic flow lines of $g_{0}$ and $g_{k-1}$ with periods $\leq t$ coincide and have the same periods. In addition the Poincaré exponents $\left(\lambda_{j}\left(g_{k-1}\right)\right)_{j=1, \ldots, L(k)}$ satisfy

$$
p(L(k), t)\left(\lambda_{1}\left(g_{k-1}\right), \ldots, \lambda_{L(k)}\left(g_{k-1}\right)\right) \neq 0 .
$$

Now we consider the polynomial

$\bar{p}_{k}\left(x_{1}, \ldots, x_{l(k)}\right)=p(L(k+1), t)\left(\lambda_{1}\left(g_{k-1}\right), \ldots, \lambda_{L(k)}\left(g_{k-1}\right), x_{1}, \ldots, x_{l(k)}\right)$

in the variables $x_{1}, \ldots, x_{l(k)}$. By definition of the polynomial $p(L(k+1), t)$ we can factorize $\bar{p}$ :

$\bar{p}_{k}\left(x_{1}, \ldots, x_{l(k)}\right)=p(L(k), t)\left(\lambda_{1}\left(g_{k-1}\right), \ldots, \lambda_{L(k)}\left(g_{k-1}\right)\right) \cdot p_{1}\left(x_{1}, \ldots, x_{l(k)}\right)$

where $p_{1}$ is a polynomial in $\mathbb{Z}\left[x_{1}, \ldots, x_{l(k)}\right]^{*}$. The first factor does not vanish by the induction hypothesis. The second factor $p_{1}$ is a product of non-trivial polynomials $q$ of the form:

$q\left(x_{1}, \ldots, x_{l(k)}\right)=\sum_{0 \leq i_{1}, \ldots, i_{l(k)}} p_{i_{1}, \ldots, i_{l(k)}}\left(\lambda_{1}\left(g_{k-1}\right), \ldots, \lambda_{L(k)}\left(g_{k-1}\right)\right) x_{1}^{i_{1}} \cdots x_{l(k)}^{i_{l(k)}}$. 
Here $p_{i_{1} \ldots i_{l(k)}}$ is a polynomial with $L(k)$ variables with degree $\leq t$ and with integer coefficients, whose absolute values are bounded by $t$. Since $q$ does not vanish identically there is a tuple $\left(j_{1}, \ldots, j_{l(k)}\right)$ with $p_{j_{1} \ldots j_{l(k)}} \neq 0$. Hence $p_{j_{1} \ldots j_{l(k)}}$ is a factor of $p(L(k), t)$, so

$$
p_{j_{1}, \ldots, j_{l(k)}}\left(\lambda_{1}\left(g_{k-1}\right), \ldots, \lambda_{L(k)}\left(g_{k-1}\right)\right) \neq 0
$$

by induction hypothesis. Therefore the polynomial $p_{1}$ is a non-trivial polynomial in the variables $x_{1}, \ldots, x_{l(k)}$. We conclude from lemma 3.3 that the invariant set

$$
\begin{array}{r}
Q_{k}:=\{P \in \operatorname{Sp}(n-1) \mid P \in \operatorname{Sp}(l(k) ; n-1) \Rightarrow \\
\left.p(L(k+1), t)\left(\lambda_{1}\left(g_{k}\right), \ldots, \lambda_{L(k)}\left(g_{k}\right), \lambda_{1}(P), \ldots, \lambda_{l(k)}(P)\right) \neq 0\right\}
\end{array}
$$

is an open and dense subset of $\operatorname{Sp}(n-1)$. We conclude from the local pertubation theorem 2.1 and from part a) of this proof: There is a metric $g_{k} \in \mathcal{U} \cap \mathcal{G}^{r}\left(\gamma_{k}, g_{k-1}, W_{k}\right)$ with

$$
P_{g_{k}}\left(\gamma_{k}\right) \in Q_{k} \cap \operatorname{Sp}(l(k) ; n-1) .
$$

Hence the periodic flow lines of period $\leq t$ of $g_{k}$ and $g_{0}$ and their periods coincide. In addition for $j \neq k$ the linearized Poincaré maps $P_{g_{k-1}}\left(\gamma_{j}\right)$ and $P_{g_{k}}\left(\gamma_{j}\right)$ coincide.

After $m(t)$ steps we obtain a metric $g=g_{m(t)}$. The periodic flow lines of period $\leq t$ and their periods of $\phi_{g_{0}}^{t}$ and $\phi_{g}^{t}$ coincide. The sequence $\Lambda_{g}(t)=$ $\left(\lambda_{1}(g), \ldots, \lambda_{n(t)}(g)\right)$ of Poincaré exponents satisfy the inequality

$$
p(n(t), t)\left(\lambda_{1}(g), \ldots, \lambda_{n(t)}(g)\right) \neq 0,
$$

which implies $g \in \mathcal{G}_{s}(t)$

Now

$$
\mathcal{G}_{s}=\bigcap_{n=1}^{\infty} \mathcal{G}_{s}(n),
$$

hence it follows from the preceding lemma:

Theorem 4.3 For a compact manifold $M$ and $2 \leq r \leq \infty$ the set of strongly bumpy metrics is a residual subset of $\mathcal{G}^{r}$.

For short one can also say: For a $C^{r}$-generic metric on a compact manifold any finite set of Poincaré exponents is algebraically independent. æ

\section{A strongly bumpy metric has infinitely many closed geodesics}

If $\gamma=\left\{\phi_{g}^{t}(v)\right\}$ is a periodic flow line ot $\phi_{g}^{t}$ of period $a$ we define the underlying closed geodesic $c=c(\gamma): S^{1}=\mathbb{R} / \mathbb{Z} \rightarrow M$ by $c(t)=\tau\left(\phi_{g}^{a t}(v)\right)$. We 
can characterize closed geodesics as critical points of the energy functional as follows: We introduce the free loop space

$$
\Lambda=\Lambda M=\left\{\sigma: S^{1} \rightarrow M \mid \sigma \text { absolutely continuous }, \int_{0}^{1} g(\dot{\sigma}, \dot{\sigma})<\infty\right\}
$$

of $M$. This is a Hilbert manifold carrying a canonical $\Phi(2)$-action. The energy functional

$$
E: \Lambda M \rightarrow \mathbb{R}, E(\sigma)=\frac{1}{2} \int_{0}^{1} g(\dot{\sigma}, \dot{\sigma})
$$

is differentiable and $\Phi(2)$-invariant. The metric $g$ induces a metric $g_{1}$ on $\Lambda M$, then the energy functional satisfies the Palais-Smale condition.

The index $\operatorname{ind}(c)$ of a closed geodesic $c$ is the index of the Hessian $d^{2} E(c)$ of $E$ at $c$, which is also called the index form. Hence the index is the dimension of a maximal subspace of $T_{c} \Lambda M$ on which the hessian is negative definite. For differentiable vector fields $X, Y$ along $c$ one has the following formula for the Hessian:

$$
d^{2} E(c)(X, Y)=\int_{0}^{1}\{g(\nabla X, \nabla Y)-g(R(X, \dot{c}) \dot{c}, Y)\} d t
$$

Here $\nabla$ denotes the covariant derivative along $c$ and $R$ denotes the Riemannian curvature tensor. The nullity null $(c)$ of a closed geodesic $c$ is the dimension of the null space of $d^{2} E(c)$ minus 1 . The nullity equals the dimension of the space of normal periodic Jacobi fields along $c$. The selfadjoint operator associated to $d^{2} E(c)$ via $g_{1}$ is of the form identity + compact operator, hence the index and the nullity are finite.

If $c$ is a closed geodesic, the iterates $c^{m}, m \geq 1, c^{m}(t)=c(m t)$ are closed geodesics, too. Two closed geodesics $c_{1}, c_{2}$ are geometrically equal resp. geometrically distinct if $c_{1}\left(S^{1}\right)=c_{2}\left(S^{1}\right)$ resp. $c_{1}\left(S^{1}\right) \neq c_{2}\left(S^{1}\right) . c$ is prime if it is not the iterate of a shorter closed curve. Hence if $c$ is a prime closed geodesic, the set of geometrically equal closed geodesics is given by $\mathbb{D}(2) \cdot c^{m}, m \geq 1$.

For the Morse theory of the energy functional the sequence $\left(\operatorname{ind}\left(c^{m}\right)\right)_{m \geq 1}$ is important. Bott introduced the following concept: Denote by $T_{c} \Lambda M_{\mathbb{C}}$ the complexified vector space of $H^{1}$-vector fields along $c$. Extend the Riemannian metric $g$ to a Hermitian form which we also denote by $g$ and extend the Riemannian curvature tensor to a complex linear tensor. Fix $z \in \mathbb{C},|z|=1$ and let

$æ$

$$
T_{c}^{z} \Lambda M:=\left\{X \in T_{c} \Lambda M_{\mathbb{C}} \mid X(1)=z X(0)\right\} .
$$

The $z$-index form

$$
H(z)(X, Y)=\int_{0}^{1}\{\langle\nabla X, \nabla Y\rangle-\langle R(X, \dot{c}) \dot{c}, Y\rangle\} d t
$$


is defined on $T_{c}^{z} \Lambda M \times T_{c}^{z} \Lambda M$. Since the corresponding selfadjoint operator $A_{c}(z)$ is again of the form identity + compact operator we can define the $z$ index $I_{c}(z)$ and the $z$-nullity $N_{c}(z)$. Here $I_{c}(z)$ is the sum of the dimension of eigenspaces of negative eigenvalues of $A_{c}(z)$ and $N_{c}(z):=\operatorname{dim} \operatorname{ker} A_{c}(z)$ if $|z| \neq 1$ and $N_{c}(1)=\operatorname{null}(c)=\operatorname{dim} \operatorname{ker} A_{c}(z)-1$. Then Bott shows in [Bo]:

Theorem 5.1 (Bott $[\mathrm{Bo}$, thm. A,C]) Let $c$ be a closed geodesic with linearized Poincaré map $P_{c} . I_{c}, N_{c}: S^{1}=\{z \in \mathbb{C}|| z \mid=1\} \rightarrow \mathbb{N}_{0}$ are the $z$-index resp. $z$-nullity. Then:

a)

$$
\operatorname{ind}\left(c^{m}\right)=\sum_{z^{m}=1} I_{c}(z) \quad, \quad \operatorname{null}\left(c^{m}\right)=\sum_{z^{m}=1} N_{c}(z)
$$

b)

$$
N_{c}(z)=\operatorname{dim} \operatorname{ker}\left(P_{c}-z \mathrm{id}\right)
$$

c) $I_{c}$ is constant in a neighborhood of points $z$ with $N_{z}(c)=0$. The splitting numbers

$$
S_{c}^{ \pm}(z) ;=\lim _{\phi \rightarrow \pm 0} I_{c}(z \exp (i \phi))-I_{c}(z)
$$

of $I_{c}$ satisfy

$$
0 \leq S_{c}^{ \pm}(z) \leq N_{c}(z)
$$

d)

$$
I_{c}(z)=I_{c}(\bar{z}), N_{c}(z)=N_{c}(\bar{z})
$$

Let $z_{j}= \pm \exp \left(2 \pi i \lambda_{j}\right), 1 \leq j \leq l-1, l \leq n, 0 \leq \lambda_{j} \leq 1 / 2$ be the eigenvalues of $P_{c}$ on $S^{1}$ resp. $\lambda_{1}<\lambda_{2}<\ldots<\lambda_{l}$ be the Poincaré exponents of $P_{c}$. Let $\lambda_{0}=0, \lambda_{l+1}=1 / 2$ and $I_{j}=I(\exp (2 \pi i \lambda))$ for $\lambda \in\left(\lambda_{j-1}, \lambda_{j}\right), 0<$ $j \leq l+1$. Let $I_{1}=I_{2}$ if $\lambda_{1}=0$ and $I_{l}=I_{l+1}$ if $\lambda_{l}=1 / 2$.

We define the difference of the splitting numbers:

$$
\epsilon(z):=S^{-}(z)-S^{+}(z) .
$$

and let $\epsilon_{j}:=\epsilon\left(z_{j}\right)$ for $j=1, \ldots, l-1$.

Corollary 5.2 [Bo, cor.1] The average index

$$
\alpha_{c}=\lim _{m \rightarrow \infty} \frac{\operatorname{ind}\left(c^{m}\right)}{m}=\int_{0}^{1} I(\exp (2 \pi i t)) d t
$$

exists and satisfies the following properties:

a)

$$
\alpha_{c}=\operatorname{ind}\left(c^{2}\right)-\operatorname{ind} c+2 \sum_{j=1}^{l} \epsilon_{j} \lambda_{j}
$$


b) If $\alpha_{c}=0$ then $\operatorname{ind}\left(c^{m}\right)=0$ for all $m \geq 1$.

c) If $c$ is hyperbolic, then

$$
\operatorname{ind}\left(c^{m}\right)=m \cdot \operatorname{ind}(c) .
$$

If all iterates of $c^{m}$ are non-degenerate i.e. if null $\left(c^{m}\right)=0$ for all $m \geq 1$ then $c$ is hyperbolic or all Poincaré exponents of $P_{c}$ are irrational. Now we want to study the equation ( 1 ) for a closed geodesic $c$ with $P_{c} \in \operatorname{Sp}(l ; n)$ and $l \geq 1$.

Bott proves in [Bo, thm. C] that the splitting numbers $S^{ \pm}(z)$ depend only on the conjugacy class of $P_{c}$ in $\operatorname{Sp}(n-1)$. We describe how the difference of the splitting numbers depends on the normal form of $P_{c}$, which we discussed in section 3 . æ

Theorem 5.3 [BTZ, 2.13] If the linearized Poincaré map $P_{c}$ has the normal form

$$
\bigoplus_{z} \bigoplus_{j=1}^{j(z)} J_{\mathbb{R}}\left(z, m_{j}, \sigma_{j}\right) .
$$

then the difference $\epsilon(z)=S^{-}(z)-S^{+}(z)$ for $z \neq \pm 1$ of the splitting numbers is given as follows:

$$
\begin{aligned}
\epsilon(z) & =\#\left\{J_{\mathbb{R}}(z, m, \sigma) \mid m \text { odd and } \sigma=-1\right\} \\
& -\#\left\{J_{\mathbb{R}}(z, m, \sigma) \mid m \text { odd and } \sigma=+1\right\}
\end{aligned}
$$

As an immediate consequence we obtain the

Corollary 5.4 If the linearized Poincaré map $P_{c}$ lies in $\operatorname{Sp}(l ; n)$ with $l \geq 1$ and if $0<\lambda_{1}<\ldots<\lambda_{l}<1 / 2$ are the Poincaré exponents of $P_{c}$ then the differences of the splitting numbers $\epsilon_{j}:=\epsilon\left(z_{j}\right)=\epsilon\left(\exp \left(2 \pi i \lambda_{j}\right)\right), j=1, \ldots, l$ satisfy:

$$
\epsilon_{j} \in\{ \pm 1\}
$$

Now we will show that a strongly bumpy metric on a simply-connected compact manifold $M$ has infinitely many geometrically distinct closed geodesics. We use a relation between the average indices of the closed geodesics of a metric with only finitely many geometrically distinct closed geodesics. The author derived this relation in [Ra1], cf. theorem $5.5 \mathrm{~b}$ ).

If $M$ is simply-connected and compact and carries a metric with only finitely many geometrically distinct closed geodesics then the sequence $\left(b_{i}(\Lambda M, F)\right)_{i \geq 1}$ of Betti numbers of the free loop space with respect to any field $F$ is bounded. This is due to a theorem by Gromoll-Meyer [GM]. Using the theory of minimal models Vigué-Poirrier/Sullivan prove in [VS] that then the rational cohomolgy algebra $H^{*}(M ; \mathbb{Q})$ is generated by a single element. 
Related results for the homology of $M$ with coefficient field of prime characteristic are proved by McCleary-Ziller [MZ] and Halperin/Vigué-Poirrier [HV].

We use the following notation: Let $T_{d, m+1}(x)$ be the truncated polynomial algebra generated by the element $x$ of degree $d$ and with the relation $x^{m+1}=0$. If $d$ is odd then $m=1$, i.e. $M$ is rationally homotopy equivalent to a $d$-dimensional sphere.

Let

$$
B(d, m)=\left\{\begin{array}{cl}
-\frac{m(m+1)}{2 d(m+1)-4} & ; d \text { even } \\
\frac{d+1}{2 d-2} & ; d \text { odd }
\end{array} .\right.
$$

For a closed geodesic $c$ we define the following metric invariant $\gamma_{c} \in$ $\{ \pm 1 / 2, \pm 1\}: \gamma_{c} \in\{ \pm 1\}$ iff $\operatorname{ind}\left(c^{2}\right) \equiv \operatorname{ind}(c) \quad(\bmod 2)$ and $\gamma_{c}>0$ iff $\operatorname{ind}(c)$ is even.

Then we have the following

Theorem 5.5 Let $M$ be a compact simply-connected manifold with a bumpy metric with only finitely many geometrically distinct closed geodesics $c_{1}, \ldots, c_{r}$. Then there are numbers $d \geq 2, m \geq 1$ with $H^{*}(M ; \mathbb{Q}) \cong T_{d, m+1}(x)$. Let $\alpha_{i}:=\alpha_{c_{i}}$ be the average indices and $\gamma_{i}:=\gamma_{c_{i}} \in\{ \pm 1 / 2, \pm 1\}$ the metric invariant defined above. Then:

a) [Hi, (6.2)], [Ra1, thm.1] There is a non-hyperbolic closed geodesic.

b) [Ra1, thm.3] The average indices $\alpha_{i}$ are positive and satisfy:

$$
B(d, m)=2 \sum_{i=1}^{r} \frac{\gamma_{i}}{\alpha_{i}} .
$$

Now we show that a metric $g \in \mathcal{G}^{*}(M)$ with only finitely many geometrically distinct closed geodesics is not strongly bumpy: $æ$

Lemma 5.6 Let $g \in \mathcal{G}^{*}(M)$ be a metric on a compact simply-connected manifold $M$ with only finitely many geometrically distinct closed geodesics $\left(c_{k}\right)_{k=1, \ldots, r}$. Let $\left(c_{k}\right)_{k=1 \ldots, m^{*}}$ be the non-hyperbolic closed geodesics with the sequence $\Lambda_{g}=\left(\lambda_{i}\right)_{i=1 \ldots, n^{*}}$ of Poincaré exponents.

Then there is a non-hyperbolic closed geodesic, i.e. $m^{*}, n^{*} \geq 1$, and there is a non-trivial polynomial $p \in \mathbb{Z}\left[x_{1}, \ldots, x_{n^{*}}\right]^{*}$ of degree $m^{*}$ or $m^{*}-1$ with

$$
p\left(\lambda_{1}(g), \ldots, \lambda_{n^{*}}(g)\right)=0 .
$$

Proof. By theorem 5.5 a) there is a non-hyperbolic closed geodesic, i.e. $m^{*}, n^{*} \geq 1$. Let $\alpha_{k}$ be the average index of $c_{k}$ which is positive by theorem $5.5 \mathrm{~b})$. Let $I_{k}=\operatorname{ind}\left(c_{k}^{2}\right)-\operatorname{ind}\left(c_{k}\right), k=1, \ldots, m^{*}$. Since $g \in \mathcal{G}^{*}(M)$ 
it follows from corollary 5.2 and corollary 5.4 that there are numbers $\epsilon_{k} \in$ $\{ \pm 1\}, k=1, \ldots, m^{*}$ with

$$
\alpha_{k}=I_{k}+2 \sum_{j=1}^{l(k)} \epsilon_{L(k)+j} \lambda_{L(k)+j} .
$$

Here $P_{c_{k}} \in \operatorname{Sp}(l(k) ; n-1)$ and $L(k):=\sum_{j=1}^{k-1} l(j)$. Let $b=d(m+1)-2$ then the topological invariant $B(d, m)$ satisfies $b \cdot B(d, m) \in \mathbb{Z}-\{0\}$. From theorem 5.5 we have

$$
B(d, m)=\sum_{k=1}^{r} \frac{\gamma_{k}}{\alpha_{k}} .
$$

Let

$$
B:=b\left(B(d, m)-\sum_{i=m^{*}+1}^{r} \frac{\gamma_{i}}{\alpha_{i}}\right)
$$

This is a rational number since for a hyperbolic closed geodesic the average index is an integer, see corollary $5.2 \mathrm{c}$ ). It follows from the equations ( 2 ) and ( 3 ) that the polynomial

$$
\begin{array}{r}
p\left(x_{1}, \ldots, x_{n^{*}}\right)=B \prod_{k=1}^{m^{*}}\left(I_{k}+2 \sum_{j=1}^{l(k)} \epsilon_{L(k)+j} x_{L(k)+j}\right) \\
-b \sum_{k=1}^{m^{*}} \gamma_{k} \prod_{s=1, s \neq k}^{m^{*}}\left(I_{s}+2 \sum_{j=1}^{l(s)} \epsilon_{L(s)+j} x_{L(s)+j}\right)
\end{array}
$$

satisfies

$$
p\left(\lambda_{1}, \ldots, \lambda_{n^{*}}\right)=0 .
$$

If $B \neq 0$ then $p$ has degree $m^{*}$, in particular $2^{m^{*}} B$ is the coefficient of $x_{1} \cdot x_{L(2)+1} \cdot \ldots \cdot x_{L\left(m^{*}\right)+1}$. If $B=0$ then $m^{*} \geq 2$ and $p$ has degree $m^{*}-1$, in particular $-2^{m^{*}-1} \cdot b \cdot \gamma_{1} \cdot \epsilon_{1} \cdot \epsilon_{L(2)+1} \cdot \ldots \cdot \epsilon_{L\left(m^{*}-1\right)+1}$ is the coefficient of $x_{1} \cdot x_{L(2)+1} \cdot \ldots \cdot x_{L\left(m^{*}-1\right)+1}$

From the definition 4.1 of a strongly bumpy metric and from the preceding lemma we conclude:

Theorem 5.7 A strongly bumpy metric on a compact simply-connected manifold has infinitely many geometrically distinct closed geodesics.

Using theorem 4.3 we obtain:

Theorem 5.8 For $2 \geq r \geq \infty$ the set of Riemannian metrics on a compact simply-connected manifold with infinitely many geometrically distinct closed geodesics contains a residual subset of the set $\mathcal{G}^{r} M$ of all Riemannian $C^{r}$ metrics.

For short one can say: For $2 \leq r \leq \infty$ a $C^{r}$-generic metric on a compact simply-connected manifold has infinitely many geometrically distinct closed geodesics. $æ$ 


\section{References}

[Ab] R.Abraham: Bumpy metrics. In: Global Analysis. Proc.Symp.Pure Math Vol. XIV Amer.Math.Soc. Providence R.I.(1970) 1-3

[An2] D.V.Anosov: On generic properties of closed geodesics (Russian) Izv.Akad.Nauk. SSSR 46 (1982) = (Engl.transl.) Math. USSR Izv. 21 (1983) 1-29

[BTZ] W.Ballmann, G.Thorbergsson \& W.Ziller: Closed geodesics on positively curved manifolds. Ann. of Math.(2) 116 (1982) 213-247

[Ba1] V.Bangert: Geodätische Linien auf Riemannschen Mannigfaltigkeiten. Jber.d.Dt.Math.-Verein. 87 (1985) 39-66

[Ba2] V.Bangert: On the existence of closed geodesics on two-spheres. Preprint IHES, Bures-sur-Yvette 1991

[Bo] R.Bott: On the iteration of closed geodesics and the Sturm intersection theory. Comm.Pure Appl.Math. 9 (1956) 171-206

[Fr] J.Franks: Geodesics on $S^{2}$ and periodic points of annulus homeomorphisms. Invent.Math. 108 (1992) 403-418

[GM] D.Gromoll \& W.Meyer: Periodic geodesics on compact Riemannian manifolds. J.Differential Geom. 3 (1969) 493-510

[Gr] K.Grove: Condition (C) for the energy integral on certain pathspaces and applications to the theory of geodesics. J.Differential Geom. 8 (1973) 207-223

[HV] S.Halperin \& M.Vigué-Poirrier: The homology of a free loop space. Pacific J.Math. 147 (1991) 311-324

[Hi] N.Hingston: Equivariant Morse theory and closed geodesics. J.Differential Geom. 19 (1984) 85-116

[Kl] W.Klingenberg: Lectures on closed geodesics. Grundlehren der math.Wiss. 230 Springer-Verlag Berlin Heidelberg New York 1978

[KT] W.Klingenberg \& F.Takens: Generic properties of geodesic flows. Math.Ann. 197 (1972) 323-334

[MZ] J.McCleary \& W.Ziller: On the free loop space of homogeneous spaces. Amer.J.Math. 109 (1987) 765-781

[Mo] J.Moser: Proof of a generalized form of a fixed point theorem due to G.D.Birkhoff. In: Springer Lect.Notes 597 (1977) 464-494 
[Ra1] H.B.Rademacher: On the average indices of closed geodesics. J.Differential Geom. 29 (1989) 65-83

[Ra2] H.B.Rademacher:Morse-Theorie und geschlossene Geodätische. Habilitationsschrift, Bonn 1991

[Ra3] H.B.Rademacher: Metrics with only finitely many isometry invariant geodesics. Math.Ann. 284 (1989) 391-407

[Ra4] H.B.Rademacher: The Fadell-Rabinowitz index and closed geodesics. to appear in: J.London Math.Soc.

[VS] M.Vigué-Poirrier \& D.Sullivan: The homology theory of the closed geodesic problem. J.Differential Geom. 11 (1976) 633-644

[Zi] W.Ziller: Geometry of the Katok examples. Ergod.Th.\& Dyn.Syst. 3 (1982) 135-157

INSTITUT FÜR MATHEMATIK

UnIVERsität AugsBurg

UNIVERSITÄTSSTR. 8

8900 Augsburg

Germany

rademacher@uni-augsburg.de 